\title{
Floyd D. Loop, MD (1936-2015)
}

\author{
Dr. Ricardo Zalaquett S. \\ Profesor Titular \\ División de Enfermedades Cardiovasculares, Facultad de Medicina \\ Pontificia Universidad Católica de Chile
}

El 11 de junio de 2015, a los 78 años de edad falleció, en Cleveland, Ohio, Floyd D. Loop, MD, lo que constituye una gran pérdida para la cardiología en general y para la cirugía cardiovascular en particular.

El Dr. Floyd Loop comenzó su entrenamiento en Cirugía Cardiovascular en la Cleveland Clinic en 1967, en los momentos en que se iniciaba en ésta la cirugía de bypass coronario por René Favaloro y Donald Effler, quienes fueron sus mentores junto a Mason Sones, el padre de la coronariografía, y se incorporó como cirujano propiamente tal a la Cleveland Clinic en 1970. No es así de extrañar que el Dr. Loop haya jugado un rol crucial en el desarrollo y refinamiento de la cirugía coronaria, lo que culminó con su publicación en el New England Journal of Medicine de 1986, la que estableció la superioridad de la arteria mamaria interna por sobre la vena safena.

Esta publicación en el NEJM se convirtió en un hito de la cardiología y la cirugía cardiovascular y referencia obligada de cualquier publicación sobre cirugía coronaria, ya que demostró que los pacientes en los cuales se anastomosó la arteria mamaria interna izquierda a la arteria descendente anterior tenían una mayor supervivencia a la vez que una menor incidencia de infarto miocárdico, re-hospitalización por causas cardíacas, re-operación y eventos cardíacos tardíos, respecto a aquellos en los que se utilizó solo la vena safena. Este beneficio fue más significativo mientras más extensa era la enfermedad coronaria. (Sorprende enterarse que este trabajo, que fue la publicación principal de enero de 1986 del NEJM y que ha sido citado hasta el momento en 1.106 oportunidades, fue inicialmente rechazado por no ser un estudio prospectivo y randomizado y sólo fue aceptado por intervención directa de Eugene Braunwald y John Kirklin!).

Floyd Loop realizó en su vida como cirujano de corazón más de 12.000 cirugías cardíacas y fue autor o coautor de más de 350 publicaciones, además de su publicación estrella de la arteria mamaria recién citada, las que abarcan todos los aspectos de la cirugía cardiovascular, destacando aquellas que llevaron a un refinamiento de la revascularización miocárdica con conductos arteriales, progresos en las técnicas de re-operación coronaria y de seguimiento de los pacientes con bypass coronario. En relación a este último aspecto, es necesario destacar que el Dr. Loop fue de importancia crucial para establecer el primer registro mundial computarizado de los resultados de la cirugía cardíaca en la Cleveland Clinic.

Floyd Loop nació en Lafayette, Indiana, en 1936. Su padre fue un médico rural que influyó importantemente en su decisión de convertirse en médico. Estudió medicina en George Washington University, en Washington, D.C., la misma universidad a la que asistió su padre, graduándose en 1962, y en la que también hizo su residencia en Ciru- 


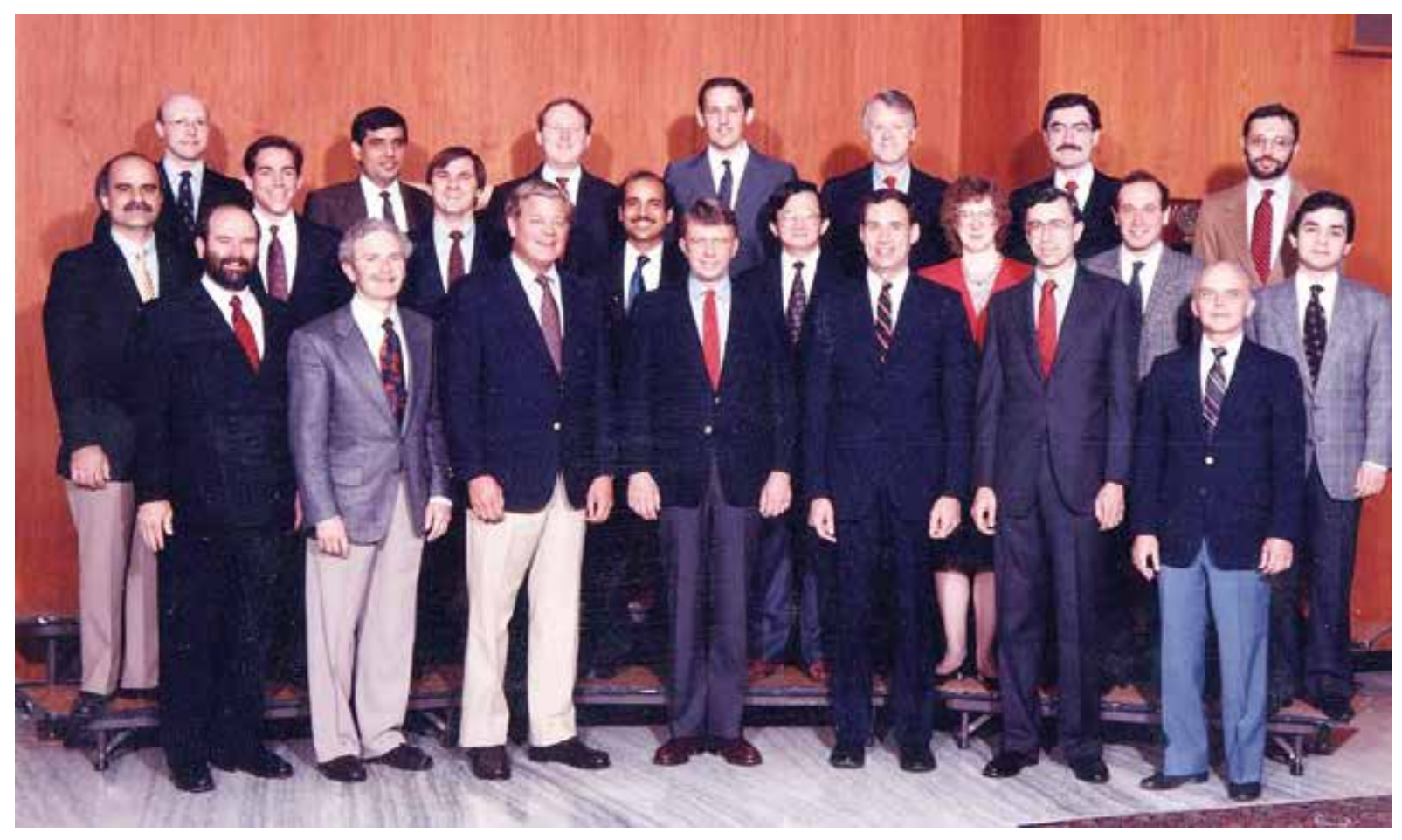

Fotografía de los miembros del Departamento de Cirugía Torácica y Cardiovascular de la Cleveland Clinic Foundation de 1988. En primera fila, de izquierda a derecha, los doctores Thomas Rice, Bruce Lytle, Paul Taylor, FLOYD D. LOOP, Delos Cosgrove, Robert Stewart y Leonard Golding. Atrás, los médicos residentes provenientes de Estados Unidos, India, Malasia, Italia, Reino Unido, Argentina, Pakistán y Chile..

gía General, con una interrupción de 2 años en la Fuerza Aérea, en la base Andrews, en la que tuvo grandes oportunidades quirúrgicas. Por consejo de Brian Blades, Jefe de Cirugía de George Washington University, y gran visionario, decidió dedicarse a la cirugía cardiotorácica, para lo cual éste le consiguió una posición como residente en la Cleveland Clinic. En 1970 ingresó como cirujano a la Cleveland Clinic, convirtiéndose en Jefe de Cirugía Torácica y Cardiovascular en 1975. Durante los próximos 14 años como jefe, el Dr. Floyd Loop logra duplicar el número de cirugías cardíacas y constituir un grupo de cirujanos cardiovasculares expertos, que van a liderar la especialidad por los años venideros, convirtiendo a la Cleveland Clinic en uno de los principales centros del mundo en cirugía cardiovascular. En 1989, Floyd Loop es nombrado CEO de la Cleveland Clinic, llevando a ésta a un crecimiento explosivo sin precedentes, posicionándola entre los mejores 10 hospitales de Estados Unidos por 10 años seguidos. Final- mente, como CEO lideró una campaña filantrópica que se tradujo en la construcción de numerosos nuevos edificios en el campus principal en Cleveland y que culminó con la fundación de una escuela de medicina, la Cleveland Clinic Lerner College of Medicine of Case Western Reserve University, la que va a tener como misión formar médicos investigadores, y que fue uno de sus proyectos más queridos. En 2004, Floyd D. Loop, MD, se retiró como CEO de la Cleveland Clinic, pero se mantuvó como consejero y mentor y se incorporó a varios boards corporativos, públicos y privados.

Floyd D. Loop, MD, tuvo gran importancia en la formación de varios cirujanos cardiovasculares chilenos, no solo como Jefe del Programa de Postítulo de Cirugía Torácica y Cardiovascular de la Cleveland Clinic, en el que estos fueron becados, sino que también a través de un contacto permanente y afectuoso en congresos y cursos, y en las visitas posteriores a la clínica. 
Referencias:

1. LOOP FD, LYTLE BW, COSGROVE DM, STEWART RW, GOORMASTIC M, WILLIAMS GW, et al. Influence of the Internal-Mamary-artery Graft on 10-Year Survival and Other Cardiac Events. N Engl J Med. 1986; 314: 1-6.

2. STONEY W. Pioneers of Cardiac Surgery. 1st ed. Van- derbilt University Press, 2008.

3. LOOP FD, LYTLE BW, COSGROVE DM, WOODS EL, STEWART RW, GOLDING LA, et al. Reoperation for Coronary Atherosclerosis. Changing Practice in 2509 Consecutive patient. AnnSurg. 1990; 212: 385-386.

(http://consultqd.clevelandclinic.org) 